\title{
Integration moderner Prozessdatenerfassung in mittleren und großen Anlagen
}

Die in den letzten 20 Jahren automatisierten Anlagen bestehen zum großen Teil aus kleineren und mittleren Anlagen. Großanlagen im Umweltbereich standen jedoch von Beginn an im strategischen Fokus international operierender Konzerne. Trotzdem waren die Vorschriften und Anforderungen an die Datenprotokollierung und -haltung bis etwa zum Jahr 2000 prinzipiell ähnlich zu mittleren und Kleinanlagen. Mit Einführung der ATVM 260 klaffte jedoch das Anforderungsprofil in der Datenerfassung zwischen den Anlagengrößen weit auseinander. Das Beispiel ACRON zeigt, wie moderne, herstellerneutrale Datenerfassungssysteme trotzdem die gesetzlichen Vorgaben problemlos erfüllen.

Die Anforderungen an eine übergreifende Datenerfassung mit Auswertung und Protokollierung für alle Anlagen eines Verbandes wurden mit der Einführung der M 260 um ein Vielfaches erhöht. Beim Oldenburg-Ostfriesischen-Wasserverband (OOWV) besteht bei Kleinanlagen aus Sicht der heutigen Technologien eine überschaubare Anforderung an die Datenhaltung. Doch bei mittleren und großen Anlagen ist allein schon das Datenaufkommen und deren Vorhaltung und Verarbeitung eine anspruchsvolle Aufgabe. Im Moment befinden sich im Bereich Abwasser 47 Kläranlagen und 1200 Pumpwerke. Der Wasserbereich zählt 15 Pumpwerke, 6 Speicherpumpwerke sowie 263 Förderbrunnen.

Mit der Änderung der Protokollierungspflicht von der H 260 auf die M 260 ergab sich ein weiteres Problem. Viele der im Einsatz befindlichen Systeme waren rein technologisch gesehen nicht mehr in der Lage, diese neuen Anforderungen zu erfüllen. In vielen Fällen wurde über Jahre versucht, die bestehenden Anlagen und Technologien zu aktualisieren, was jedoch meist an einer falschen technologischen Strategie scheiterte. Hier seien die vielen Lösungen mittels SQL-Server erwähnt, die bis heute fast alle abgelöst wurden. Deren Ablösung zu Recht erfolgte, da einer der Kernpunkte der Datenhaltung darauf basiert, dass alle Daten

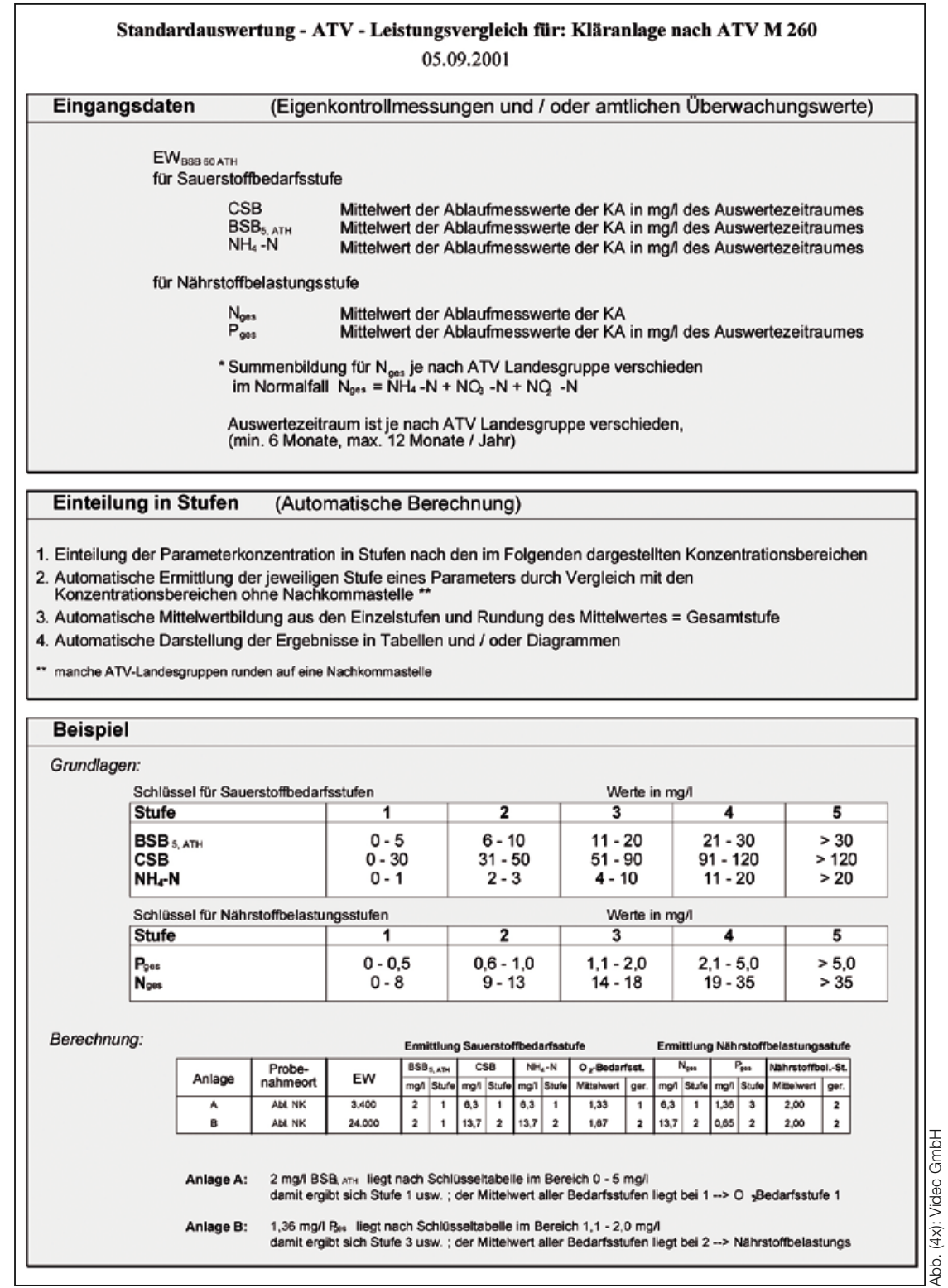

Abb. 1: ATV-M 260-Leistungsvergleich.

für mindestens 10 Jahre vorzuhalten sind. Damit sind die Rohwerte aus den Automatisierungsanlagen gemeint, die in unterschiedlicher Dichte und Geschwindigkeit in die Datenbankgeschrieben werden müssen. Dort werden die Rohdaten berechnet, in unterschiedliche Stufen verdichtet und der Auswertung zur Verfügung gestellt.

\section{Datensicherheit in jeder Anlagenstruktur}

Noch aufwendiger war die Bewältigung der Anforderungen an Protokollierung und grafische Auswertung. Die Berichte und Protokolle sind um ein Vielfaches komplexer geworden, der Zugriff auf die Werte mit dem grafischen Werkzeug auf wenige Se- 
kunden festgelegt. Mit dieser Festlegung waren nahezu alle Visualisierungen/Leitsysteme und Datenbanken vor nur sehr schwer zu lösende Aufgaben gestellt.

Für diese übergreifenden Anwendungen bewährte sich das Konzept des Produktes ACRON. Kernpunkt ist die Beibehaltung aller Rohwerte im System - auch für die gesicherten Daten. Diese Lösung ermöglicht - auch bei Datenauslagerung jederzeit ein Reproduzieren der historisch tatsächlichen Zustände.

Für den schnellen Zugriff auf Langzeitdaten wird mit berechneten Daten (Mittelwerten) gearbeitet. Diese werden in gesonderte Archive geschrieben.

Dies bedeutet, dass bei einer Auswertung stets auf den optimalen Datenbereich zugegriffen wird und somit die Frontends schnelle Informationen an den Bediener liefern. Funktionell klingt diese Lösung sehr kompliziert, für den Anwender bedeutet dies jedoch nur wenige Klicks in der Oberfläche, und schon ist die gewünschte, individuelle Auswertung parat.

Prinzipiell wird auf Anlagen, die der Größe des OOWV entsprechen, mit redundanten HMI-, SCADA- oder Leitsystemen gearbeitet. Das bedeutet, dass die Struktur dieser Systeme dem des Archivierungssystem entsprechen muss. Um eine vielfältige Peripherie an Systemen bedienen zu können, müssen sowohl Datenhaltung als auch Datenerfassung redundant auslegbar sein.

Zurückgebrochen auf den Systemeinsatz einer Archivierung in einem Verband (beispielsweise OOWV) sind diese Anforderungen enorm breit gestreut:

- Durchgängigkeit vom kleinen Einplatzsystem bis herauf $\mathrm{zu}$ redundanten Mehrplatzsystemen.

- Unterschiedliche Anforderungen dieser Anlagen seitens der Normung (hier ATV-M 260) mit einer strukturellen Datendurchgängigkeit. Diese wird wichtig, wenn eine zentrale Datenhaltung mit einem Benchmarking der Anlagen in Aussicht steht.

\section{Individuelle und einfache Bedienbarkeit}

Beim OOWV oder bei entsprechend größeren Kläranlagen sind stets Systeme mit mehreren Arbeitsplätzen erwünscht. Hier bietet sich eine Client-Server-Architektur an, die das Arbeiten aus den unterschiedlichen Bereichen (Labor, Leitstand etc.) gewährleistet. Zusätzlich können Datenzugriffe nur für spezifische Abteilungen

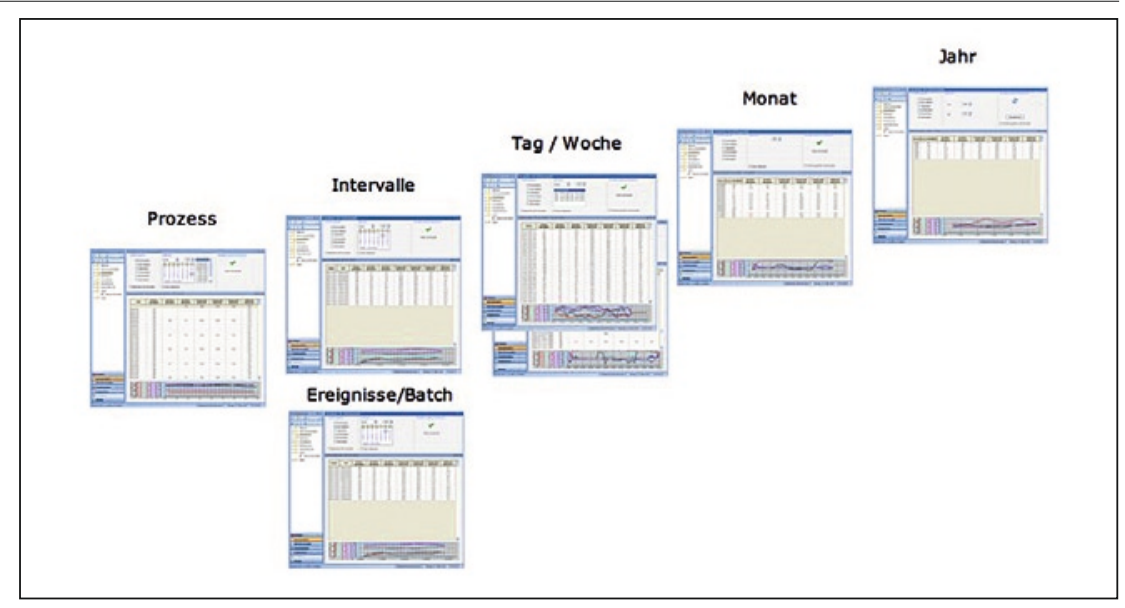

Abb. 2: Für den schnellen Zugriff auf Langzeitdaten arbeitet ACRON mit berechneten Daten (Mittelwerten), die in gesonderte Archive geschrieben werden.

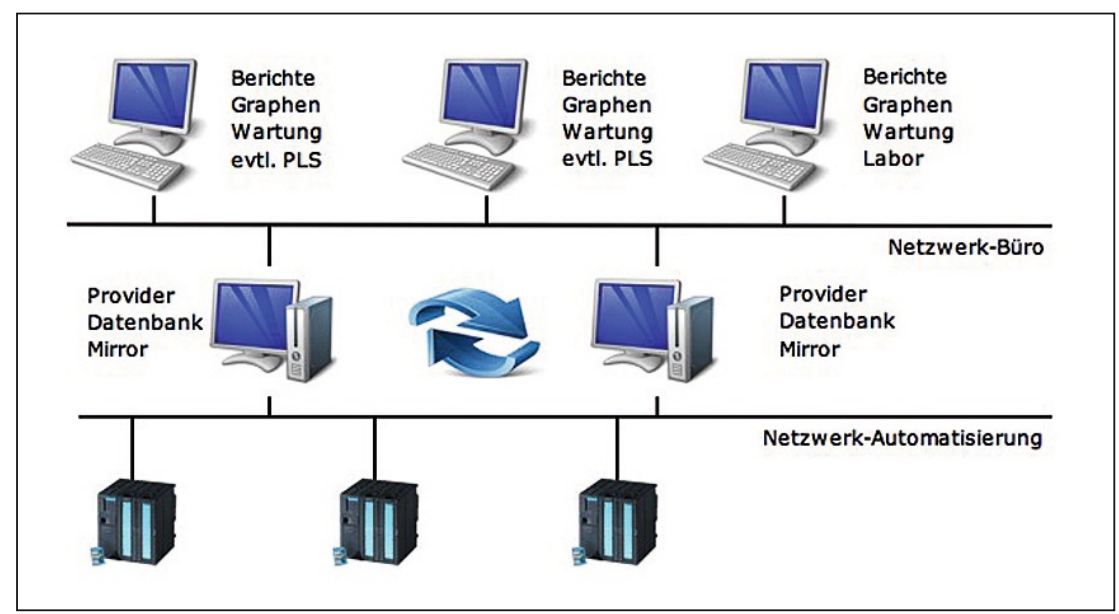

Abb. 3: Mit ACRON ist eine bis 8-fach redundante Datenbank möglich.

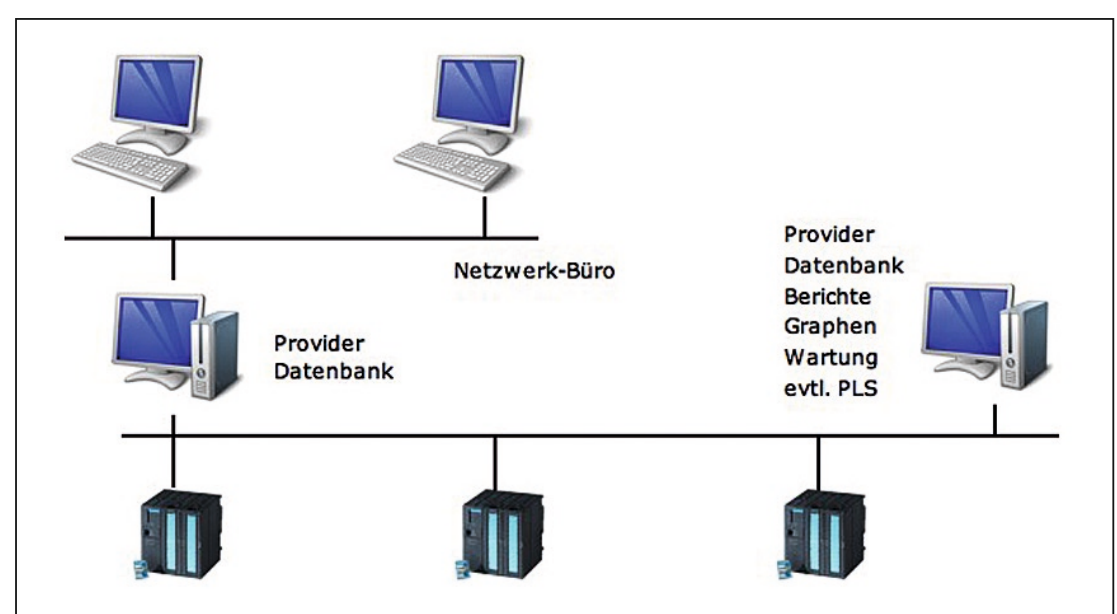

Abb. 4: Eine flexible Client-Server-Architektur bietet viele Netzwerk-Möglichkeiten mit ACRON (1 bis $n$ Arbeitsplätze).

bzw. Personen zur Verfügung gestellt werden. Trotzdem bleibt die Datenstruktur sicher.

Zentrales Thema bei ACRON ist die einfache Handhabung für das tägliche Arbeiten. Die Software ist so konzipiert, dass sowohl bei unterschiedlichem Ausbildungsstand des Betriebspersonals als auch für Mitarbeitergenerationen, die nicht mit der EDV groß geworden sind, ein intuitives Umgehen mit dem System möglich ist. Alle für die Bereiche Wasser- und 
Abwasser notwendigen Informationen stehen dem Anwender mit wenigen Bedieneingaben zur Verfügung. Ein Expertenmodus lässt das Arbeiten in unterschiedlichen Ebenen zu. Beim OOWV sind alle Funktionen und Optionen entsprechend den verantwortlichen Personen freigeschaltet.

\section{Berücksichtigung der "Total Costs of Ownership“}

Da ACRON keine individuelle Programmierung für den OOWV ist, sondern eine übergreifende Produktlösung, sind alle Lizenzen problemlos zu warten und einfach in das Gesamtkonzept zu integrieren. Selbst bei Zukäufen von Anlagen ist es für den OOWV in sehr kurzer Zeit möglich, die bestehenden Strukturen der Anlagen ohne große Investitionen in das vorhandene Konzept zu integrieren. Für alle Lizenzen wurde ein Software-Wartungsvertrag abgeschlossen, um immer den optimalen technologischen Stand zu gewährleisten.

Ein weiterer wichtiger Punkt für den Einsatz von ACRON beim OOWV war die Verfügbarkeit mehrerer Integratoren für die Implementierung und Systempflege. Hier wollte man sich nicht von einem Unternehmen abhängig machen und einen gesunden Wettbewerb im Bieterkreis gewährleisten. Dieser Punkt konnte mit der breiten Palette von zertifizierten ACRONSystempartnern voll erfüllt werden.

\section{Fazit}

Zusammengenommen ergibt sich eine Mischung aus hohen Anforderungen mit einer durchgängigen Struktur und einer einfachen Bedienung bei einer breiten Funktionalität. Für den Einsatz in der Wasser- und Abwasserbranche kommen nur Systeme mit überzeugendem Preis-Leistungs-Verhältnis infrage - besser lässt sich die Verwendung von ACRON beim OOWV nicht beschreiben.

\section{Informationen:}

Industrial Automation

Hr. Klaus Lussnig

Tel. +43-650-5877644

Fax +43-512-2199213586

lussnig@industrial-automation.at

www.industrial-automation.at 